\title{
PRINCIPIOS QUE INFORMAN LA REGULACIÓN DE LOS CRITERIOS LEGALES PARA ESTABLECER EL RIESGO DE CONFUSIÓN ENTRE \\ MARCAS
}

\section{Principles that support the regulation of the legal factors to determine the likelihood of confusion between trademarks}

\author{
Carlos Cornejo Guerrero* \\ Universidad Nacional Mayor de San Marcos \\ Lima, Perú
}

RESUMEN: Uno de los temas fundamentales dentro de la disciplina del Derecho de Marcas es el referido a los criterios para establecer el riesgo de confusión entre tales signos distintivos. La utilidad de toda la disciplina del Derecho de Marcas, sus principios, conceptos e instituciones, la sofisticación de sus reglas y su desarrollo orgánico tienen como propósito último y principal, el solucionar los conflictos que se presentan en la práctica mercantil sobre posible confusión entre marcas ya registradas y las que se solicitan a registro o en el supuesto de infracciones de marcas. Los criterios legales, jurisprudenciales o doctrinarios que establecen el riesgo de confusión entre marcas no deben ser aplicados mecánicamente, sino que hay que ajustar su utilización a las especificidades del caso concreto. En el presente artículo se busca esclarecer y explicar los principios o premisas de

Profesor principal de la Facultad de Derecho y Ciencia Política de la Universidad Nacional Mayor de San Marcos, correo electrónico: <carloscornejoguerrero@yahoo.com>.

Artículo recibido el 15 de febrero de 2015 y aprobado el 24 de abril de 2015. 
los que parte la formulación de dichos criterios según la normativa legal peruana, de modo que los criterios mencionados se apliquen con justeza y cumplan el objetivo de solucionar adecuadamente los conflictos entre marcas.

PALABRAS CLAVE: principios, criterios, riesgo de confusión, marcas, propiedad intelectual.

ABSTRACT: One of the fundamental issues within the discipline of the Trademark Law is the question of the criteria for determining the risk of confusion between such trademarks. The usefulness of the entire discipline of Trademark Law, its principles, concepts and institutions, the sophistication of its rules and its organic development has, as ultimate and primary purpose, solving conflicts that are presented in the commercial practice about possible confusion between marks already registered and those requested to register in the case of trademark infringement. Legal, jurisprudential or doctrinal criteria that establish the risk of confusion between marks, should not be applied mechanically, but must be adjusted to use in the specific case. This article seek to clarify and explain the principles or premises from which part the formulation of the criteria according to Peruvian legislation, to fairness aplication of them, and will give a clean solution between trademarks conflicts.

KEY WORDS: principles, factors, criteria, likelihood of confusion, trademarks, intellectual property.

\section{INTRODUCCIÓN}

Uno de los temas fundamentales dentro de la disciplina del Derecho de Marcas es el referido a los criterios para establecer el riesgo de confusión entre tales signos distintivos. Podemos decir, sin temor a equivocarnos, que la utilidad de toda la disciplina del Derecho de Marcas, todos sus principios, conceptos e instituciones, la sofisticación de sus reglas y su desarrollo orgánico, tienen como propósito último y principal, el solucionar los conflictos que se presentan en la práctica mercantil sobre posible confusión entre marcas ya registradas y las que se solicitan a registro'.

1 Dependiendo del sistema de adquisición del derecho de marcas vigente en cada país, el referido conflicto también podrá presentarse entre marcas en uso y otras marcas cuyo uso y/o registro sea posterior. De otro lado, en nuestro país asimismo, pueden presentarse 
El Derecho de Marcas se ha elaborado para solucionar este problema; para no permitir que se afecte un derecho de marcas ya constituido, sea porque se otorgue otro que entre en conflicto con él o porque se ampare el uso de una marca incompatible con la marca registrada. En este sentido, la idea de protección del derecho constituido no es una simple declaración, sino que es un concepto que adquiere la categoría de principio jurídico; este principio puede tener efectos en la solución concreta de conflictos entre marcas. El Derecho de Marcas comporta un sub-sistema jurídico, que forma parte de otro sub-sistema más amplio que es el de los signos distintivos, el que a su vez está integrado a un sistema mayor denominado Propiedad Industrial.

En dicho sistema se aplican principios, conceptos e instituciones que preservan su armonía y que están alimentados por la realidad de los negocios; es por ello que un método o aproximación adecuados para el estudio de este tema es partir de la observación de la realidad, particularmente del análisis de la jurisprudencia y de las nuevas herramientas publicitarias que utilizan las empresas de hoy en día, en donde la marca ocupa un lugar privilegiado. Este enfoque, el del análisis de las instituciones con la ayuda de la jurisprudencia y de la revisión de la práctica mercantil diaria, es la que guiará nuestro trabajo.

El estudio de los criterios para determinar la confundibilidad de las marcas es importantísimo, porque es la respuesta del Derecho de Marcas a ciertos actos típicos de competencia indebida. En algunos países, los criterios a aplicarse para los casos de confundibilidad entre marcas se han establecido en la Ley². La elaboración de estos criterios y su plasmación en la Ley, no se ha producido de un día para otro, sino que por lo general, fue la Jurisprudencia la que primero dejó sentado los conceptos básicos aplicables a los casos presentados en la realidad, luego la doctrina desarrolló y sistematizó estos conceptos en base a las decisiones jurisprudenciales, y por último la Ley los depuró y los consagró positivamente.

casos de infracción del derecho de marcas que se siguen en un procedimiento especial diferente al procedimiento de oposición a la solicitud de registro de marca; en estos supuestos, igualmente tendrá que evaluarse la proximidad entre la marca registrada y aquella con la que se estaría cometiendo el acto de infracción.

2 Como ha sido el caso del Perú. Como un ejemplo de los países que no cuentan con una plasmación legislativa de los criterios aplicables al cotejo marcario está la República Argentina, donde se ha desarrollado una creativa jurisprudencia. NúÑEZ (2002) fascículo $n^{\circ} 9$. 
La primera norma jurídica peruana que consigna los criterios para determinar la confundibilidad entre las marcas es el Decreto Ley $\mathrm{n}^{\circ} 26.017$, el cual es antecedente inmediato del Decreto Legislativo $n^{\circ}$ 823, publicado el 24 de Abril de $1996^{3}$. En realidad, el Decreto Legislativo $n^{\circ} 823$ se ha elaborado con base en el Decreto Ley 26.017, de 1992, mejorando algunos aspectos puntuales, pero manteniendo su idea de fondo. Asimismo, el Decreto Legislativo $\mathrm{n}^{\circ} 1.075^{4}$, actualmente vigente, tiene prácticamente el mismo texto que el del Decreto Legislativo ${ }^{\circ} 823$.

Las normas antedichas han consagrado criterios que ya habían sido elaborados por la doctrina nacional y extranjera, y que eran ampliamente conocidos y utilizados desde antaño. La plasmación de estos criterios en la Ley, además de confirmar la justeza de los desarrollos doctrinales en la materia, proporciona una guía útil y adecuada para resolver los conflictos entre marcas. Se ha legislado con la suficiente flexibilidad, para que en el examen de confundibilidad, se pueda atender a las particularidades del caso concreto, sin que queden desvirtuados los principios generales sobre los que descansa esta disciplina.

Si bien es cierto este artículo tratará sobre los principios que informan la regulación de los criterios legales para establecer el riesgo de confusión entre marcas tomando como base la normatividad peruana, nada impide que sea

3 Decreto Legislativo $n^{\circ}$ 823, de 1996. La primera obra peruana en la que se categorizan y analizan los criterios para establecer la confundibilidad entre marcas es: Cornejo (1992), del autor de este trabajo. La obra se publicó antes que en el Perú se produjera el establecimiento en la Ley, de los criterios para determinar la confundibilidad entre marcas.

4 Decreto Legislativo que aprueba Disposiciones Complementarias a la Decisión n 486 de la Comisión de la Comunidad Andina que establece el Régimen Común sobre Propiedad Industrial. El artículo 45 del Decreto Legislativo ${ }^{\circ} 1.075$, de 2008, de tiene el texto siguiente:

"Artículo 45.- Determinación de semejanza.

A efectos de establecer si dos signos son semejantes y capaces de inducir a confusión y error al consumidor, la Dirección competente tendrá en cuenta, principalmente los siguientes criterios:

a) la apreciación sucesiva de los signos considerando su aspecto de conjunto, y con mayor énfasis en las semejanzas que en las diferencias;

b) el grado de percepción del consumidor medio;

c) la naturaleza de los productos o servicios y su forma de comercialización o prestación; respectivamente;

d) el carácter arbitrario del signo, su uso, publicidad y reputación en el mercado; y

e) si el signo es parte de una familia de marcas". 
de utilidad cuando dichos criterios han sido elaborados por la jurisprudencia o doctrina; por otro lado, el trabajo también será útil cuando en el país de que se trate no exista un desarrollo particular sobre el tema.

Antes de entrar al estudio de los criterios legales, jurisprudenciales o doctrinarios que establecen el riesgo de confusión entre marcas, es importante esclarecer cuáles son las principios o premisas de los que parte la formulación de dichos criterios, así como cuál es la naturaleza del examen de confundibilidad entre marcas a realizarse por el órgano competente ${ }^{5}$, razón por la cual nos hemos avocado al tratamiento de este tema.

\section{EN EL EXAMEN COMPARATIVO, EL JUZGADOR DEBE SITUARSE LO MÁS CERCA POSIBLE A LAS MISMAS CONDICIONES Y CIRCUNSTANCIAS EN QUE ESTARÍA EL PÚBLICO CONSUMIDOR PERTINENTE, AL MOMEN- TO EN QUE ADQUIERE DETERMINADO PRODUCTO O SERVICIO}

El principio básico que informa los criterios legales para examinar la confundibilidad entre marcas consiste en que, en tal examen, es indispensable que el juzgador se sitúe lo más cerca posible a las mismas condiciones y circunstancias en que estaría el público consumidor pertinente, al momento en que adquiere determinado producto o servicio. Los criterios plasmados en la ley, no son otra cosa que guías o pautas probadas por la ciencia y la experiencia, tendientes a que el examen de confundibilidad reproduzca o se aproxime a la situación real en la que se vería incurso el consumidor. En el examen de confundibilidad entre marcas y específicamente en la aplicación de los criterios concretos para establecer si hay riesgo de confusión, es fundamental tener en mente este principio básico.

Un criterio fundamental que se desprende de este principio es que en el examen comparativo el juzgador debe ponerse en la situación del consumidor medio. La noción de consumidor medio es un estándar jurídico que sólo

En el Perú, los criterios para establecer la confundibilidad entre marcas han sido plasmados en la Ley y los casos sobre conflictos entre marcas causados por este motivo se conocen en primer término en sede administrativa (Dirección de Signos Distintivos del Indecopi, primera instancia administrativa y Sala de Propiedad Intelectual del Tribunal del Indecopi, segunda Instancia que agota la vía administrativa. Las Resoluciones del Tribunal son impugnables ante el Poder Judicial, dentro del procedimiento contenciosoadministrativo. Sin embargo, hay que tener en cuenta que no en todos los países se sigue este sistema para la resolución de estos conflictos. En la República Argentina, por ejemplo, sólo es el Poder Judicial quien conoce y decide los casos de conflicto surgidos por un posible riesgo de confusión entre marcas. 
puede determinarse según las especificidades del caso concreto; sin embargo para aproximarse a su concepto, muchas veces se realiza la clasificación siguiente:

Consumidor diligente: Es aquel consumidor analítico, observador, de especial cultura y que está bien informado.

Consumidor medio: Es aquel consumidor que no es muy diligente, pero tampoco muy distraído.

Consumidor distraído: Es aquél consumidor poco observador, que no pone cuidado en la atención a las cosas o asuntos y que no está bien informado.

A veces ha surgido la discusión de si el criterio aplicable para determinar el riesgo de confusión entre marcas o el existente para resolver los conflictos en sede de competencia desleal, debe ser el concerniente al del consumidor medio o al del consumidor razonable; estimándose que para los casos de propiedad industrial (como son los de conflictos entre marcas) debe aplicarse la noción de consumidor medio; en cambio, para los conflictos propios de la competencia desleal debe aplicarse la noción del consumidor razonable. Así se ha establecido en la legislación y en la jurisprudencia en el Perú.

A veces se estima que la noción de consumidor razonable implica un grado más alto de diligencia que el consumidor medio y también se ha indicado que la noción de consumidor razonable refiere a un consumidor que está a mitad de camino entre el consumidor medio y el consumidor diligente. Nosotros disentimos de estas posiciones y pensamos que no hay mucha diferencia entre la noción de consumidor medio y la de consumidor razonable. La noción de consumidor razonable se comenzó a utilizar en el Perú por vía jurisprudencial administrativa ${ }^{6}$, señalándose que esta correspondía a aquél consumidor que actuara con una diligencia ordinaria. Un consumidor que actúa con una diligencia ordinaria, es decir la diligencia esperada según una situación determinada, no es muy diferente a un consumidor medio que no es totalmente diligente, pero tampoco distraído.

${ }_{6}$ Tribunal del Instituto Nacional de Defensa de la Competencia y de la Propiedad Intelectual - INDECOPI. 


\section{LA ELECCIÓN DE LOS CRITERIOS APLICABLES DEBE HACERSE SE- GÚN LAS ESPECIFICIDADES DEL CASO CONCRETO}

Otro principio importante es el de que la elección de los criterios aplicables debe hacerse según las especificidades del caso concreto. Este principio tiene dos consecuencias importantes. La primera es la consideración -que se desprende inclusive de la misma Ley- que los criterios expresamente señalados en ella, son los que principalmente se tomarán en cuenta, pero no son necesariamente los únicos. Es decir, la lista de criterios indicados del artículo $45^{\circ}$ al artículo $49^{\circ}$ del Decreto Legislativo $n^{\circ} 1.075$, no constituye una lista taxativa sino meramente enunciativa ${ }^{7}$.

Aún cuando de la lectura del primer párrafo del artículo $45^{\circ}$ del Decreto Legislativo $\mathrm{n}^{\circ} 1.075$ se desprenda que se da mayor importancia a los criterios

$7 \quad$ El artículo 45 del Decreto Legislativo ya fue transcrito en la nota 5. Los artículos 46 al 49 del mismo cuerpo legal tienen el texto siguiente:

"Artículo 46.- Signos denominativos Tratándose de signos denominativos, en adición a los criterios señalados en el artículo 45 de este Decreto Legislativo, se tendrá en cuenta lo siguiente:

a) la semejanza gráfico-fonética;

b) la semejanza conceptual; $y$,

c) si el signo incluye palabras genéricas y/o descriptivas, se realizará el análisis sobre la palabra o palabras de mayor fuerza distintiva.

Artículo 47.- Signos figurativos Tratándose de signos figurativos, en adición a los criterios señalados en el artículo 45 de este Decreto Legislativo, se tendrá en cuenta lo siguiente:

a) Si las figuras son semejantes, si suscitan una impresión visual idéntica o parecida.

b) Si las figuras son distintas, si evocan un mismo concepto.

Artículo 48.- Signos mixtos Tratándose de signos mixtos, formados por una denominación y un elemento figurativo, en adición a los criterios señalados en los artículos 45.46 y 47 del presente Decreto Legislativo se tendrá en cuenta lo siguiente:

a) la denominación que acompaña al elemento figurativo;

b) la semejanza conceptual; $y$

c) la mayor o menor relevancia del aspecto denominativo frente al elemento gráfico, con el objeto de identificar la dimensión característica del signo.

Artículo 49.- Semejanza conceptual Tratándose de un signo denominativo y uno figurativo, se tendrá en consideración la semejanza conceptual. Tratándose de un signo denominativo y uno mixto, se tendrán en cuenta los criterios señalados en los artículos 45 y 47 de este Decreto Legislativo. Tratándose de un signo figurativo y uno mixto, se tendrán en cuenta los criterios señalados en los artículos 47 y 48 del presente Decreto Legislativo.

En los tres supuestos serán igualmente de aplicación los criterios señalados en el artículo 45 del presente Decreto Legislativo." 
consignados expresamente por dicha norma, ello no niega que en el caso concreto sea también importante la aplicación de otros criterios. Ello está plenamente justificado, en vista de la riqueza de matices y particularidades que puede presentar la realidad, que no harían aconsejable una limitación de los criterios. Los criterios consignados expresamente por la Ley, no constituyen en modo alguno, una camisa de fuerza para el juzgador; como hemos mencionado, la propia Ley deja en claro que tales criterios no son los únicos, sino solamente aquellos que por su importancia, deben ser considerados principalmente.

Algunos criterios que no están contemplados en la norma y que dado el caso podrían invocarse, son por ejemplo el de la coexistencia pacífica de marcas, el de que mayor significación para la comparación tienen los elementos característicos o más distintivos de las marcas, o el de hay que tener en cuenta los informes de sondeo de opinión o encuestas entre los consumidores potenciales de los productos a ser distinguidos con la marca, etc.

También debemos considerar en relación a este aspecto, que los criterios legales para establecer el riesgo de confusión entre marcas han sido formulados teniendo en mente signos tradicionales o típicos; recurriendo a la clasificación clásica de marcas que las divide en nominativas, figurativas y mixtas. Ello ha generado que nuevos tipos de marcas permitidos por la actual Legislación, e inclusive signos que eran permitidos por la Legislación anterior, como las marcas tridimensionales, no encuentren una regulación específica en este aspecto.

De esto puede sobrevenir la creación de nuevos criterios aplicables a estas clases especiales de marcas, o por lo menos, el perfeccionamiento de los antiguos. Un ejemplo de este fenómeno es el que se presenta cuando se trata de establecer la confundibilidad de dos marcas tridimensionales que consisten en la forma del producto. Un criterio que no encontramos plasmado en la Legislación, pero que tiene mucha justificación es el de que en estos casos el ámbito de protección de la marca tridimensional registrada, sólo puede llegar a impedir la utilización de exactamente la misma forma tridimensional registrada o una que sea casi la misma. Es decir, en el examen de confundibilidad entre estas marcas, se considerará que la protección que brinda una marca tridimensional es más reducida, por lo que no se rechazará el registro de una marca tridimensional solicitada, cuando pese a que presente similitud con la registrada, no se trate de la misma forma ni de una muy cercana a ella.

La segunda consecuencia del principio de elección de los criterios aplicables, según las especificidades del caso concreto, es que no necesariamente podrán siempre aplicarse todos los criterios consagrados en la Ley para la 
solución del caso. Imagínese por ejemplo que existiera un conflicto entre dos marcas nominativas, siendo que ninguna de ellas perteneciera a una familia de marcas; entonces, no habría forma de aplicar el referido criterio aún cuando esté consignado expresamente en el literal e) del artículo 45 del Decreto Legislativo $\mathrm{n}^{\circ}$ 1.075. Es decir, como bien dice la ley, la Dirección competente tendrá en cuenta estos criterios, pero lógicamente sólo entrarán a tallar cuando el caso concreto pueda adecuarse al supuesto de hecho previsto en la norma.

\section{DEBE HACERSE UNA LIBRE PONDERACIÓN DEL PESO DE LOS CRI- TERIOS APLICABLES A UN CASO PARTICULAR}

Además, tenemos el principio de la libre ponderación del peso de los criterios aplicables a un caso particular. El rol de los criterios no es el dar una solución mecánica a los casos, sino más bien proporcionar una guía de los aspectos que deben ser considerados al momento de evaluar si dos marcas son confundibles o no. Es indispensable hacer una verdadera evaluación del caso concreto, conjugando de la mejor manera los criterios aplicables.

No puede dejar de observarse, en vista de la naturaleza del examen de confundibilidad entre marcas, que siempre habrá un cierto grado de subjetividad al momento de decidir en un sentido u otro. Los criterios señalados buscan -en la medida de lo posible- reducir ese grado de subjetividad y de incertidumbre que se produce normalmente por interpretaciones o evaluaciones que no se ajusten a parámetros ya aceptados; sin embargo, siempre serán las particularidades del caso concreto, a las que habrá que poner atención especial para una correcta decisión.

Refiriéndonos en conjunto, al principio de la elección de los criterios según las especificidades del caso concreto y al de la libre ponderación del peso de los criterios aplicables en un caso particular, se debería considerar por ejemplo, que no todos los criterios legales serían aplicables al momento de comparar dos marcas, además que no todos tienen necesariamente la misma jerarquía y que algunas veces habrán criterios que no han sido consignados en la Ley, pero que deben aplicarse en atención al caso particular.

Para establecer el riesgo de confusión en los Estados Unidos de América, las Cortes aplican varios factores que se derivan del caso "Polaroid Corp. v. Polarad Electronics Corp."; se entiende que estos factores sirven como guía y no 
todos ellos pueden ser de utilidad en un caso determinado ${ }^{8}$. Es interesante ver la similitud de estos factores con los criterios que se aplican en nuestra legislación.

\section{ES IMPORTANTE TENER EN CUENTA LA CLASIFICACIÓN DE LOS CRITERIOS EN GENERALES Y ESPECIALES}

El principio referido a la clasificación de los criterios en generales ${ }^{9}$ y especiales es indispensable para no olvidar todas las variables que deben aplicarse al momento de juzgar un caso. Se entiende que los criterios generales se aplican a cualquier clase de conflicto entre marcas, los criterios especiales únicamente a los conflictos derivados de marcas del mismo tipo, según la clasificaciónn anotada que divide a las marcas en denominativas, figurativas y mixtas. Esta regulación servirá de guía para establecer cuáles son los criterios aplicables, por ejemplo, entre dos o más marcas denominativas, dos o más marcas figurativas, marcas denominativas versus marcas mixtas, etc. Los criterios especiales se aplican, según los tipos de marca de que se trate, adicionalmente a los criterios generales.

La formulación de los criterios en base a esta diferenciación permite, además, aplicar en lo posible, los criterios generales a relativamente nuevos tipos de marcas, sin que la no existencia de criterios dirigidos específicamente a ellas, se constituyan en un obstáculo para la decisión.

8 Citado por STIM (2000) p.127. Los factores referidos son los siguientes: strength of the senior's user mark, similarity of the marks, similarity of the products or services, likelihood that the senior user will bridge the gap, the junior user's intent in adopting the mark, evidence of actual confusion, sophistication of the buyers and quality of the junior user's products or services.

9 Es posible encontrar otras clasificaciones de los criterios. Por ejemplo en la República Argentina, se habla de reglas o principios y de circunstancias adjetivas para el cotejo marcario. Estas reglas o principios y circunstancias adjetivas han sido desarrolladas por la jurisprudencia. Así en la Cámara Nacional de Apelaciones en lo Civil y Comercial Federal, Pfizer Inc. y Finadiet S.A. (1998, causa n 3369/98) sobre cese de oposición al Registro de Marca, se señala: "Antes que nada voy a recordar algunos principios que a los fines de la confrontación marcaria, ha sentado la jurisprudencia del Tribunal [...]". Asimismo, más adelante se indica: "[...] En definitiva, como en todos los conflictos marcarios, habrá de ponderarse el escenario en el cual se ambienta la contienda o "las circunstancias adjetivas de la causa", sin que quepa limitar la decisión a un desnudo cotejo de los signos". 


\section{EN LA APLICACIÓN DE LOS CRITERIOS PROPIOS DEL EXAMEN DE CONFUNDIBILIDAD ENTRE MARCAS Y, EN GENERAL, EN EL DEL EXAMEN DE REGISTRABILIDAD, SE EVALUARÁN ASPECTOS DE HECHO COMO DE DERECHO, Y NO SÓLO ESTOS ÚLTIMOS}

El presente principio está referido a la naturaleza del examen de confundibilidad entre marcas y -en general- al examen de registrabilidad. Este consiste en que, en la aplicación de los criterios, se evaluarán aspectos tanto de hecho, como de derecho, y no sólo estos últimos.

En relación a este punto, la Resolución n 0329-2004 del Instituo Nacional de Defensa de la Competencia y de la Protección de la Propiedad Intelectual ${ }^{10}$, recaída en el expediente $\mathrm{n}^{0}$ 185700-2003 ${ }^{11}$, que versa sobre solicitud de registro de marca de producto, presentada por Industria de Calcado S.A.C. - ICALSAC (Perú), señala lo siguiente: Finalmente, en el procedimiento de registro de marcas, no existe la posibilidad ni la obligación de parte de la Autoridad de determinar concretamente si el público realmente aprecia el signo como proveniente de un determinado origen empresarial. En el examen de distintividad, se trata de una cuestión de derecho, que se decide en base a hechos y experiencias generales sin que sea necesario realizar investigaciones extensas (por ejemplo encuestas de consumidores). Sin embargo, sí se requiere que la decisión de la Autoridad se base en apreciaciones concretas; por lo que suposiciones teóricas, especulaciones, conjeturas, no son suficientes para determinar que un signo no es distintivo ${ }^{12}$.

Asimismo, en cuanto al examen de confundibilidad ente marcas, la Resolución no 0112-2005/TPI-INDECOPI, del 10 de febrero del 2005, recaída en el expediente $n^{\circ} 180182-2003$, sobre solicitud de registro de marca de producto Ola, para distinguir leche y productos lácteos, productos a base de yogurt, postres, batidos de leche, bebidas licuadas en las que predomina la

10 En adelante, "INDECOPI".

11 En la indicada solicitud se pedía el registro de la marca de producto constituida por la figura tridimensional de un calzado para niño en cuya suela se aprecian ruedas y diversas figuras; la parte lateral se encuentra compuesta por el diseño de ruedas; la parte frontal se encuentra compuesta por el diseño de llantas y diversas líneas; conforme al modelo, para distinguir calzado, prendas de vestir y sombrerería, de la clase 25 de la Nomenclatura Oficial.

12 En Resolución № 1326-2005/TPI-INDECOPI, p. 151 y 152, aparece un criterio similar. Véase comentario en el punto V.1.3.1, titulado: "Aproximación a la percepción efectiva del consumidor". 
leche (smoothies), clase 29, presentada por Unilever N.V. (Reino de los Países bajos), señala:

\section{CERTIFICADO N ${ }^{0} 5.647$}

"Con relación a lo manifestado por el solicitante -en el sentido que, en la práctica, las marcas de la opositora distinguen únicamente conservas de pescado, por lo que su coexistencia en el mercado no es susceptible de inducir al público consumidor a confundir un producto por otro o asociar el origen empresarial de los mismos-, la sala conviene en señalar que existe diferencia entre el registro y el uso de un signo. Así, el riesgo de confusión en derecho de marcas no es una cuestión de hecho sino de derecho y se establece basándose en dos elementos: la similitud entre los productos que distinguen cada uno de los signos y la semejanza entre los signos -en ambos casos de acuerdo a cómo se encuentren registrados o solicitados- tomando además en consideración la distintividad de los signos. En tal sentido, los productos que la marca distinga en la práctica o la forma particular como el titular del signo comercialice sus productos en el mercado no son relevantes a efectos de determinar el riesgo de confusión entre los signos".

Es pertinente destacar en relación a los extractos de las resoluciones transcritas, que el examen de registrabilidad, o específicamente el examen de confundibilidad entre marcas, no necesariamente consiste en una cuestión de puro Derecho, sino que-dicho examen-requiere el análisis de cuestiones de Derecho conjuntamente con una evaluación de los hechos, conforme a la prueba actuada.

También debe tenerse presente que muchas veces puede ser necesario, para que el juzgador forme su convicción, el contar con estudios de encuestas de consumidores o estudios de mercadeo. En la decisión sobre la registrabilidad o confundibilidad, entran en juego variables complejas referidas a la realidad del mercado y a la percepción del consumidor medio, para cuyo conocimiento, los estudios técnicos correspondientes son muy útiles y a veces indispensables.

El juzgador debe valerse de todos los medios permitidos a su alcance, para garantizar una decisión justa, aprovechando y no desechando las herramientas técnicas disponibles, cuando sea conveniente. 


\section{SE PODRÁN ACEPTAR ACUERDOS DE COEXISTENCIA DE MARCAS, CUANDO NO SE AFECTE EL INTERÉS GENERAL DE LOS CONSUMIDORES}

Por otro lado, la aplicación del examen de registrabilidad y específicamente la apreciación de la posible confundibilidad entre marcas, no queda descartada necesariamente, en el supuesto que hubiera un acuerdo de coexistencia entre los titulares de las marcas en conflicto. Así, el artículo 56 del Decreto Legislativo $n^{\circ} 1.075$, establece que las partes en un procedimiento podrán acordar la coexistencia de signos idénticos o semejantes siempre que, en opinión de la autoridad competente, la coexistencia no afecte el interés general de los consumidores.

La Resolución n 1934-2003/TPI-INDECOPI, del 2 de Octubre del 2003, emitida en el Expediente ${ }^{\circ}$ 176997-2003, sobre solicitud de registro de la marca de producto constituida por la denominación bellezza escrita en letras características, la palabra toallas escrita en letras características y la representación estilizada de un sol y tres líneas ondulantes, todo en los colores azul, turquesa, amarillo y anaranjado; conforme al modelo, para distinguir toallas de materias textiles, de la clase $n^{\circ} 24$ de la Nomenclatura Oficial:

\section{Acuerdo de coexistencia}

\subsection{MARCO CONCEPTUAL}

Las marcas como instrumentos de identificación y diferenciación de los productos o servicios hacen posible la producción en masa y el desarrollo industrial y comercial de una sociedad.

Las marcas coadyuvan a la transparencia en el mercado al permitirle a su titular individualizar -entre la multiplicidad de productos o servicios que se encuentran en el mercado- los productos o servicios que ofrece. De esta forma puede diferenciar sus productos o servicios de los de sus competidores y crear y asegurarse una clientela.

La marca permite a los consumidores diferenciar inequívocamente los productos o servicios que son ofrecidos en el mercado, y en base a esta diferenciación asociar los productos o servicios con una determinada calidad.

El interés del público consumidor en que exista la debida diferenciación entre los competidores radica en la posibilidad de identificar los productos o servicios que ha probado y con los cuales ha quedado satisfecho, sin posibilidad de error alguno. Este interés normalmente coincide con el interés privado del industrial, del comerciante o del prestador de servicios de cautelar 
su derecho exclusivo al uso de los signos que distinguen a los productos o servicios que ofrecen, por ser el primer interesado en que si un consumidor ha quedado satisfecho con su producto o servicio lo adquiera nuevamente sin que, por la similitud de los signos, el consumidor termine adquiriendo un producto o servicio de un competidor.

Puede darse, sin embargo, el caso de que dos titulares lleguen a un acuerdo económico de coexistencia de sus respectivas marcas pero que el riesgo de confusión subsista, con lo que se privaría al consumidor de su derecho a elegir libremente entre los productos del mercado, de acuerdo a su origen empresarial, además el consumidor puede verse expuesto a adquirir un producto o servicio que no posea la calidad o alguna otra característica esperada. Para impedir lo anterior, el artículo 158 del Decreto Legislativo $n^{\circ} 823$ (1) establece expresamente que las partes en un procedimiento podrán acordar la coexistencia de marcas similares para productos o servicios de la misma clase, siempre que, en opinión de la oficina administrativa competente, la coexistencia no afecte negativamente el interés general de los consumidores.

Si bien la Decisión $n^{\circ} 486$ no incluye ninguna norma que expresamente regule este supuesto, el Tribunal de Justicia de la Comunidad Andina se ha pronunciado sobre el tema. En el Proceso No 41-IP-99 -en una sentencia expedida durante la vigencia de la Decisión 344 (2) - en relación al caso de una solicitud de registro de una marca similar a otra ya registrada, señaló que pese a existir una autorización del titular cuyo registro ya está consolidado; necesariamente se tiene que considerar el interés del consumidor quien puede llegar a verse confundido con la presencia de dos marcas similares en el mercado, más aún si ellas se encuentran destinadas a proteger productos de la misma clase internacional; y si, además, las marcas confrontadas han sido solicitadas para todos los productos o servicios comprendidos en la correspondiente clase (3). En tal sentido, concluyó que en estos casos el administrador al momento de valorar estas circunstancias, en su decisión deberá velar que las marcas a confrontarse no produzcan confusión en el mercado. Precisó que el acuerdo puede facilitar el registro, pero la autoridad nacional puede tomar su decisión en contra, no conduciendo el acuerdo necesariamente a un registro (4).

En este sentido, frente a acuerdos de coexistencia o cartas de consentimiento para el registro de un signo distintivo idéntico o similar a uno ya registrado, se evaluará la posibilidad de aceptar dichos acuerdos siempre que no afecten el interés general de los consumidores; a su vez se podrá tomar en consideración, el hecho de estar frente a casos en que las partes intervinientes formen parte de un mismo grupo empresarial o tengan la condición de sucursales, ello debidamente acreditado en el procedimiento correspondiente; es 
decir, que tengan vínculos económicos, al grado que el público consumidor no se vea inducido a confusión en el mercado.

\subsection{Aplicación al caso concreto}

Tejidos San Jacinto S.A. presentó un acuerdo de coexistencia de marcas celebrado entre las empresas Inversiones Comindustria S.A. y Tejidos San Jacinto S.A.

En dicho documento se señala lo siguiente:

- "Inversiones Comindustria S.A. otorga consentimiento a Tejidos San Jacinto S.A. para que registre y use la marca "bellezza" y logotipo, únicamente para distinguir toallas, excluyendo los demás productos de la clase comprendidos en el registro de la marca registrada a favor de Inversiones Comindustria S.A. (certificado $N^{\circ}$ 73971), particularmente ropa de cama y de mesa, y asume que no levantará ninguna objeción para el uso y registro respecto al producto indicado.

- Tejidos San Jacinto S.A. se compromete a realizar el registro únicamente para toallas de materias textiles, particularmente de felpa, comprometiéndose a hacer referencia a su razón social en sus productos, con lo cual evitaría la posibilidad de cualquier riesgo de confusión.

- El acuerdo y consentimiento es válido para los registros de marcas que realicen ambas empresas en países pertenecientes a la Comunidad Andina y países latinoamericanos y centroamericanos, incluyendo México, de tal manera que los registros comprendan sólo los productos mencionados.

Respecto a dicho documento, cabe señalar que ha sido firmado por el señor Geri Ciabatti Salocchi en representación de Inversiones Comindustria S.A. y el señor Ramón Ricardo Rázuri Ramírez en representación de Tejidos San Jacinto S.A., y las firmas han sido legalizadas por el notario Jaime Murguía Cavero.

No obstante lo anterior, en aras del derecho de los consumidores (derecho reconocido en "el artículo 158 del Decreto Legislativo 823) a una debida diferenciación de los servicios que ofrecen los competidores para que puedan tomar decisiones de compra racionales basadas principalmente en calidad y precio, se tendrá que realizar el examen de confundibilidad entre los signos en conflicto, para determinar si corresponde o no aceptar el acuerdo de coexistencia presentado". 


\section{Citas de la Resolución:}

(1) "Artículo 158.- Las partes en un procedimiento podrán acordar la coexistencia de signos idénticos o semejantes siempre que, en opinión de la Oficina competente, la coexistencia no afecte el interés general de los consumidores.

(2) La Decisión 344 tampoco incluía norma alguna que expresamente regulara este supuesto.

(3) Gaceta Oficial del Acuerdo de Cartagena No 542 del 8 de marzo de 2000, p. 15.

(4) Gaceta Oficial del Acuerdo de Cartagena No 542 (nota 3), p. 19".

Como vemos, es posible el registro de marcas que deriven de un acuerdo de coexistencia pacífica entre las partes; sin embargo, ello es posible siempre que no se afecte el interés general de los consumidores.

El punto importante es determinar cuándo se afecta o no el interés general de los consumidores. Pensamos que si las marcas son semejantes, sólo pueden existir condiciones especialísimas en el que esta afectación no genere riesgo de confusión y en consecuencia no se afecte el interés general de los consumidores. Ello podría ocurrir, si entre las partes se ha acordado dirigirse a segmentos de mercados distintos y perfectamente delimitados. Ello se plasmaría, por ejemplo, si una de las partes vende sus productos directamente al público individual y tiene prohibido venderlos a grandes consumidores (hospitales, clínicas, centros escolares, universidades, institutos, etc.). También es posible aceptar estas marcas si sus titulares pertenecen a un mismo grupo empresarial o si las empresas tienen algún vínculo organizativo o económico.

\section{CONCLUSIONES}

Como podemos apreciar, la aplicación de los criterios legales para establecer el riesgo de confusión entre marcas es un asunto complejo que tiene siempre un margen de subjetividad. No obstante y justamente por ello, es muy útil conocer los principios sobre las cuales descansa la aplicación de tales criterios, para aplicarlos correctamente de una manera creativa, flexible y razonable.

Los criterios son pautas o guías que sirven para la solución de casos, que están construidos en base a la experiencia y práctica jurídico-mercantil y que nunca deben aplicarse como una camisa de fuerza en la decisión. Los principios que hemos estudiado ayudan a aplicar dichos criterios con tino y acierto. 


\section{BIBLIOGRAFÍA CITADA}

Bertone, Luis y Cabanellas, Guillermo (1989): Derecho de Marcas (Buenos Aires, Editorial Heliasta S.R.L.) Tomos I y Tomo II, 602 pp.

Breuer, Pedro (1937): Tratado de Marcas de Fábrica y Comercio (Buenos Aires, Librería y Casa Editora de Jesús Menéndez) 665 pp.

Cornejo, Carlos (2007): Derecho de Marcas (2ºdición, Lima, Cultural Cuzco) 565 pp.

(1992): Derecho de Marcas (Lima, Cultural Cuzco) 256 pp.

FerRándiz, José (2007): Jurisprudencia sobre Propiedad Industrial, Publicidad y Derecho de la Competencia (Madrid, La Ley, Grupo Wolters Kluwer) 1374 pp.

INDECOPI (1996): Compilación Legislativa de Propiedad Intelectual (Lima, INDECOPI) 420 pp.

NúÑEZ, Javier (2002): "La similitud confusionista en materia marcaria: reglas jurisprudenciales sobre cotejo de los signos", J.A. 2002-III, fascículo n 9.

Otamend, Jorge (2002): Derecho de Marcas (4 Edición, Buenos Aires, Lexis Nexis Abeledo Perrot) 395 pp.

Universidad De Buenos Aires (2007): Propiedad Industrial Facultad de Derecho, VIII Curso Intensivo de Post-grado invierno, materiales de lectura (Buenos Aires, Universidad de Buenos Aires).

Stim, Richard (2000): Trademark Law (Canada, West Legal Studies - Thomson Learning) 293 pp.

\section{NORMAS JURÍDICAS CITADAS}

Decisión $n^{\circ}$ 486, de la Comisión de la Comunidad Andina, establece un régimen común sobre propiedad industrial. Gaceta Oficial del Acuerdo de Cartagena, 19 de septiembre de 2000. Disponible en: <http://www. indecopi.gob.pe/repositorioaps/0/10/par/leg_norsupra/decis486comcomand.pdf $>$, fecha de consulta: 2 de febrero de 2015 .

Decreto Legislativo no 823 (Perú), Ley de Propiedad Industrial. Diario Oficial El Peruano, 24 de abril de 1996. 
Decreto Legislativo $n^{\circ} 1.075$ (Perú), queaprueba Disposiciones Complementarias a la Decisión n 486 de la Comisión de la Comunidad Andina, que establece el Régimen Común sobre Propiedad Industrial. Diario Oficial El Peruano, 28 de junio de 2008.

Decreto Ley no 26.017 (Perú), Ley General de Propiedad Industrial. Diario Oficial El Peruano, 12 de diciembre de 1992. Derogado por la Cuarta Disposición Final del Decreto Legislativo $n^{\circ}$ 823. Diario Oficial El Peruano, 24 de abril de 1996.

\section{JURISPRUDENCIA CITADA}

Cámara Nacional De Apelaciones Federal En Lo Civil y Comercial, Pfizer Inc. Contra Finadiet S.A, Sentencia de la Sala $n^{\circ} 2$ (1998): 9 de setiembre de 2004, Causa n³369/98.

Instituto Nacional de Defensa de la Competencia y de la Protección de la Propiedad Intelectual (INDECOPI), solicitud de registro de la marca de producto constituida por la denominación BELLEZZA, TOALLAS, clase 24 de la Nomenclatura Oficial (2003): 2 de Octubre de 2003, Resolución n ${ }^{\circ}$ 1934-2003/TPI-INDECOPI, Expediente nº 176997-2003.

solicitud de registro de marca de producto, presentada por Industria de Calcado S.A.C. - ICALSAC, Perú (2004), Resolución n 0329-2004-INDECOPI, expediente No 185700-2003.

solicitud de registro de marca de producto OLA, para distinguir leche y productos lácteos, bebidas licuadas en las que predomina la leche (smoothies), clase 29, Unilever N.V del Reino de los Países bajos (2005): 10 de febrero de 2005, Resolución n 0112-2005/TPI-INDECOPI, expediente $\mathrm{n}^{\circ}$ 180182-2003.

solicitud de registro de marca, Unión de Cervecerías Peruanas Backus y Johnston S.A.A, Perú (2005), Resolución no 1326-2005/TPIINDECOPI, expediente $\mathrm{n}^{\circ}$ 195126-2003.

En Resolución No 1326-2005/TPI-INDECOPI, p. 151 y 152, aparece un criterio similar. Véase comentario en el punto V.1.3.1, titulado: “Aproximación a la percepción efectiva del consumidor". 